\title{
Integrals of Products of Laguerre Polynomials
}

\author{
By R. D. Lord
}

In a recent note in this journal Gillis and Weiss [1] have evaluated as a finite sum, and found a recurrence relation for, the integral

$$
C_{r s t}=\int_{0}^{\infty} e^{-x} L_{r}(x) L_{s}(x) L_{t}(x) d x
$$

which gives the coefficients in the expansion

$$
L_{r}(x) L_{s}(x)=\sum C_{r s t} L_{t}(x),
$$

where $L_{n}(x)$ denotes the Laguerre polynomial,

$$
L_{n}(x)=\sum_{\nu=0}^{n}(-1)^{\nu}\left(\begin{array}{l}
n \\
\nu
\end{array}\right) \nu^{r} / r !
$$

This attracted my interest since about ten years ago I considered the more general integral of the product of generalized Laguerre polynomials

$$
\int_{0}^{\infty} e^{-x} x^{\beta} L_{n_{1}}^{\left(\alpha_{1}\right)}\left(a_{1} x\right) L_{n_{2}}^{\left(\alpha_{2}\right)}\left(a_{2} x\right) \cdots L_{n_{m}}^{\left(\alpha_{m}\right)}\left(a_{m} x\right) d x
$$

with the object of finding cases which simplified. I tried methods which I had used with the corresponding problem for Hermite polynomials [2], but met with little success. But the special case (1) is easily dealt with as follows.

The generating function for Laguerre polynomials is

$$
\sum_{r=0}^{\infty}(-1)^{r} L_{r}(x) u^{r}=\frac{1}{1+u} \exp \left(\frac{x u}{1+u}\right)
$$

and hence that for the product of three polynomials is

$$
\begin{aligned}
\sum_{r=0}^{\infty} \sum_{s=0}^{\infty} \sum_{t=0}^{\infty}( & -1)^{r+s+t} L_{r}(x) L_{s}(x) L_{t}(x) u_{1}^{r} u_{2}^{s} u_{3}^{t} \\
= & {\left[\left(1+u_{1}\right)\left(1+u_{2}\right)\left(1+u_{3}\right)\right]^{-1} \exp x\left(\frac{u_{1}}{1+u_{1}}+\frac{u_{2}}{1+u_{2}}+\frac{u_{3}}{1+u_{3}}\right) . }
\end{aligned}
$$

On multiplying by $e^{-x}$ and integrating from 0 to $\infty$, we get

(5) $\sum_{r=0}^{\infty} \sum_{s=0}^{\infty} \sum_{t=0}^{\infty}(-1)^{r+s+t} C_{r s t} u_{1}^{r} u_{2}^{s} u_{3}^{t}=\left(1-u_{2} u_{3}-u_{3} u_{1}-u_{1} u_{2}-2 u_{1} u_{2} u_{3}\right)^{-1}$

as the generating function for $C_{r s t}$. If we write the right hand side of (5) as

$$
\sum\left(u_{2} u_{3}+u_{3} u_{1}+u_{1} u_{2}+2 u_{1} u_{2} u_{3}\right)^{\nu}
$$

we immediately see that $C_{r s t}$ is an integer with the sign $(-)^{r+s+t}$. Further expansion of each term by the multinomial theorem gives Watson's formula [3]

Received 18 March 1960. 


$$
C_{r s t}=(-2)^{r+s+t} \sum_{n} 2^{-2 n} \frac{n !}{(n-r) !(n-s) !(n-t) !(r+s+t-2 n) !},
$$

where in the summation all factorials have non-negative arguments.

If we transpose the right hand side of (5) and equate coefficients of $u_{1}{ }^{r} u_{2}^{8} u_{3}{ }^{t}$, we have

$$
C_{r s t}=C_{r, s-1, t-1}+C_{r-1, s, t-1}+C_{r-1, s-1, t}+2 C_{r-1, s-1, t-1},
$$

which is the recurrence formula with simplest coefficients, especially as the right hand side can be treated as the sum of five terms. Even though all suffixes vary, it probably provides the quickest way of computing all values of $C_{r s t}$ up to a given set of $r, s, t$. For some machines at least, it may well give the quickest way for calculating a given $C_{r s t}$, and it provides an easy method for desk-machine computation when $r, s$ and $t$ are small. For computations by other methods it provides a simple check. Other checks may be obtained by giving $u_{1}$ special values in (5) and equating coefficients of $u_{2}{ }^{8} u_{3}{ }^{t}$. Putting $u_{1}=-1$, we get

$$
\sum_{r} C_{r s t}=1
$$

given by Gillis and Weiss; putting $u_{1}=-\frac{1}{2}$, we get

$$
\sum_{r} 2^{s+t-r} C_{r s t}=\left(\begin{array}{c}
s+t \\
s
\end{array}\right) \text {. }
$$

Mathematics Department,

Royal College of Science and Technology,

Glasgow, Scotland

1. J. Gillis \& G. Weiss, "Products of Laguerre polynomials," Math. Comp. (MTAC), v. 14,1960 , p. $60-63$.

2. R. D. LoRD, "Some integrals involving Hermite polynomials," London Math. Soc., Jn., v. 24, 1949, p. 101-112.

3. G. N. WATson, "A note on the polynomials of Hermite and Laguerre," London Math. Soc., Jn., v. 13, 1938, p. 29.

\section{The Evaluation of Certain Probability Integrals}

\section{By Irwin Greenberg}

A problem which often arises in the field of probability and statistics is the following:

Assume that there are $n$ independent stochastic processes and that the $k$ th process has an output distribution $f_{k}\left(x_{k}\right)$. The probability that the $j$ th process yields a higher output than any of the others is

$$
P_{j}=\int_{-\infty}^{\infty} f_{j}\left(x_{j}\right) \prod_{\substack{k=1 \\ k \neq j}}^{n} \int_{-\infty}^{x_{j}} f_{k}\left(x_{k}\right) d x_{k} d x_{j} .
$$

In certain special cases, equation (1) is easily integrated; for example, if the various $f_{k}\left(x_{k}\right)$ are all uniform or exponential distributions.

Received April 5, 1960; revised May 25, 1960. 